\title{
Coloração total em grafos potência de ciclo
}

\author{
Alesom Zorzi ${ }^{1 *}$ \\ Orientadores: Celina M. H. de Figueiredo ${ }^{1}$, Raphael C. S. Machado ${ }^{2,3}$ \\ ${ }^{1}$ COPPE/PESC - Universidade Federal do Rio de Janeiro (UFRJ) \\ Rio de Janeiro - RJ - Brasil \\ ${ }^{2}$ Instituto de Computação - Universidade Federal Fluminense (UFF) \\ Niterói - RJ - Brasil \\ ${ }^{3}$ Programa de Pós-Graduação em Metrologia e Qualidade - Inmetro \\ Duque de Caixias - RJ - Brasil \\ \{alesom, celina\}@cos.ufrj.br, rcmachadodinmetro.gov.br
}

\begin{abstract}
We present a power of cycle graph composition technique that leads to significant contributions to the state of the art of the total coloring problem in the class, providing evidence for a conjecture open 13 years ago, even with recent studies addressing the problem. The results achieved also improve the state of the art concerning the famous Total Coloring Conjecture, proposed independently by Behzad and Vizing 55 years ago.
\end{abstract}

Resumo. Apresentamos uma técnica de composição de grafos potência de ciclo que utilizamos para gerar contribuições significativas para o estado da arte do problema da coloração total na classe, gerando evidências para a validade de uma conjectura aberta há 13 anos, mesmo com estudos recentes abordando o problema. Os resultados deste trabalho também corroboram com uma famosa conjectura que foi proposta independentemente por Behzad e Vizing há 55 anos.

\section{Introdução}

O número cromático total $\chi_{T}(G)$ é o mínimo número de cores necessárias para colorir vértices e arestas de um grafo $G$ de forma que elementos adjacentes recebam cores distintas. Determinar o parâmetro $\chi_{T}(G)$ de um dado grafo $G$ é uma tarefa desafiadora e inspirou a famosa Conjectura da Coloração Total (TCC), proposta independentemente em dois trabalhos fundamentais há 55 anos [Behzad 1965, Vizing 1965], que estabelece que para todo grafo simples $G$ com grau máximo $\Delta(G)$ tem-se $\chi_{T}(G) \leqslant \Delta(G)+2$, e permanece aberta. Grafos são Tipo 1 se $\chi_{T}(G)=\Delta(G)+1$, e são Tipo 2 se $\chi_{T}(G)=\Delta(G)+2 q$.

Um grafo potência de ciclo $C_{n}^{k}$ (para $n \geqslant 3$ e $k \geqslant 1$ inteiros) é um grafo obtido a partir de um ciclo sem cordas $C_{n}$ adicionando arestas entre todos os vértices à distância de no máximo $k$. Os grafos potência de ciclo têm sido extensivamente estudados na literatura, principalmente quando tratamos de problemas de coloração. Problemas clássicos, como coloração de vértices e coloração de arestas, foram totalmente resolvidos nos grafos potência de ciclo. Surpreendentemente, apesar de avanços para valores específicos de $n$ e $k$, o problema da coloração total permanece aberto para a classe [Campos e de Mello 2007, Almeida et al. 2014].

*Financiado parcialmente pelo CNPq (bolsa 831003/1999-5) 
A notável Conjectura 1.1 estabelece que todo grafo potência de ciclo $C_{n}^{k}$ com $n>3(k+1)$ é Tipo 1 e, consequentemente, o número de grafos Tipo 2 é finito.

Conjectura 1.1 ([Campos e de Mello 2007]) Um grafo potência de ciclo $C_{n}^{k}$ que não é um ciclo nem um grafo completo (i.e. $2 \leqslant k<\lfloor n / 2\rfloor$ ) é: Tipo 2, se $n$ é ímpar e $n<3(k+1)$; Tipo 1, caso contrário.

Uma coloração harmônica é uma condição necessária para que um grafo seja Tipo 1. Os grafos $C_{n}^{k} \operatorname{com} n$ ímpar e $n<3(k+1)$ não possuem coloração harmônica e, portanto, não podem ser Tipo 1 [Campos e de Mello 2007]. A Tabela 1 ilustra o estado da arte dos problemas da TCC e da Coloração Total.

\begin{tabular}{|c|c|c|c|c|c|c|c|c|c|c|c|c|c|c|}
\hline k n & $2 k+1$ & $2 \mathrm{k}+2$ & $2 k+3$ & $2 k+4$ & $2 k+5$ & $2 k+6$ & $2 k+7$ & $\ldots$ & $n$ ímpar < $3(k+1)$ & $r(2 k+1)$ & $r(2 k+1)+k$ & $\ldots$ & n par & n ímpar $\geq 3(k+1)$ \\
\hline 2 & $\Delta+1$ & $\Delta+1$ & $\Delta+2$ & $\Delta+1$ & $\Delta+1$ & $\Delta+1$ & $\Delta+1$ & $\ldots$ & $\mathrm{C}$ & $\Delta+1$ & $\Delta+1$ & $\ldots$ & $\Delta+1$ & $\Delta+1$ \\
\hline 3 & $\Delta+1$ & $\Delta+1$ & $\Delta+2$ & $\Delta+1$ & $\Delta+2$ & $\Delta+1$ & $\Delta+1$ & $\ldots$ & $\mathrm{C}$ & $\Delta+1$ & $\Delta+1$ & $\ldots$ & $\Delta+1$ & $\Delta+1$ \\
\hline 4 & $\Delta+1$ & $\Delta+1$ & $\Delta+2$ & $\Delta+1$ & $\Delta+2$ & $\Delta+1$ & $\Delta+1$ & $\ldots$ & $\mathrm{C}$ & $\Delta+1$ & $\Delta+1$ & $\ldots$ & $\Delta+1$ & $\Delta+1$ \\
\hline 5 & $\Delta+1$ & $\Delta+1$ & $\Delta+2$ & TCC & $\Delta+2$ & TCC & 0 & $\ldots$ & C & $\Delta+1$ & TCC & $\ldots$ & TCC & 0 \\
\hline$\ldots$ & $\ldots$ & $\ldots$ & $\ldots$ & $\ldots$ & $\ldots$ & $\ldots$ & $\ldots$ & $\ldots$ & $\ldots$ & $\ldots$ & $\ldots$ & $\ldots$ & $\ldots$ & $\ldots$ \\
\hline k & $\Delta+1$ & $\Delta+1$ & $\Delta+2$ & TCC & $\Delta+2$ & TCC & $\mathrm{O}$ & $\ldots$ & C & $\Delta+1$ & TCC & $\ldots$ & TCC & 0 \\
\hline
\end{tabular}

Tabela 1. O estado da arte dos problemas da TCC e da Coloração Total na classe dos grafos potência de ciclo $C_{n}^{k}$. A entrada $\Delta+1$ representa os grafos que são Tipo 1; $\Delta+2$ representa os grafos que são Tipo 2 ; TCC representa que a TCC foi mostrada para o grafo, entretanto a classificação entre Tipo 1 e Tipo 2 ainda permanece aberta; $O$ símbolo $O$ representa os grafos em que a TCC está aberta; e $C$ corresponde aos grafos $C_{n}^{k} \operatorname{com} n$ ímpar e $n<$ $3(k+1)$ que não possuem uma coloração harmônica. Todas as entradas $\Delta+2$ e algumas entradas $O$ na tabela também satisfazem a condição $C$.

No presente trabalho mostramos, com base em dois grafos potência de ciclo $C_{n_{1}}^{k}$ e $C_{n_{2}}^{k}$ com uma coloração total Tipo 1, uma coloração total Tipo 1 para um grafo $C_{n}^{k}$, com $n=n_{1}+n_{2}$. Esta técnica, que possibilitou grande parte dos resultados presentes no trabalho, foi chamada de composição e só é possível se os grafos $C_{n_{1}}^{k}$ e $C_{n_{2}}^{k}$ forem compatíveis. A composição de grafos potência de ciclo é uma técnica que pode ser aplicada a outros grafos potência de ciclo e pode possivelmente ser generalizada para outras classes de grafos. Fazendo uso da composição de potência de ciclo, mostramos que todo grafo potência de ciclo com $k$ par e com um número suficientemente grande de vértices $\left(n \geqslant 4 k^{2}+2 k\right)$ é Tipo 1 .

Além disso, classificamos todos os grafos potência de ciclo $C_{n}^{k}$ com $k=3 \mathrm{e}$ $k=4$ e também obtemos um limitante para que os grafos potência de ciclo $C_{n}^{k}$ com $k=5$ e $k=7$ sejam Tipo 2. Ademais, apresentamos um algoritmo para gerar uma coloração harmônica equilibrada para os grafos potência de ciclo $C_{n}^{k} \operatorname{com} n$ par ou $n$ ímpar e $n \geqslant 3(k+1)$, em um passo necessário para a construção de uma coloração total equilibrada Tipo 1 .

Teorema 1.2 (Corolário 3.15 da dissertação) Um grafo potência de ciclo $C_{n}^{k}$ que não é um ciclo nem um grafo completo (i.e. $2 \leqslant k<\lfloor n / 2\rfloor$ ) é: não harmônico (e, portanto, não pode ser Tipo 1), se né ímpar e $n<3(k+1)$; harmônico, caso contrário.

Todos os resultados supramencionados estão ilustrados na Tabela 2, que quando comparada com a Tabela 1 mostra o impacto do trabalho. Nas seções seguintes descrevemos, de forma resumida, os principais resultados obtidos pelo candidato durante o seu mestrado. 


\begin{tabular}{|c|c|c|c|c|c|c|c|c|c|c|c|c|}
\hline$n$ & $2 k+1$ & $2 k+2$ & $2 k+3$ & ... & $n$ ímpar $<3(k+1)$ & $4 k+1$ & $4 k+2$ & $4 k+3$ & $4 k+4$ & $4 k+5$ & $\ldots$ & $n \geq 4 k^{2}+2 k$ \\
\hline 3 & $\Delta+1$ & $\Delta+1$ & $\mathrm{NH}$ & $\ldots$ & $\mathrm{NH}$ & $\Delta+1$ & $\Delta+1$ & $\Delta+1$ & $\Delta+1$ & $\Delta+1$ & $\ldots$ & $\Delta+1$ \\
\hline 4 & $\Delta+1$ & $\Delta+1$ & $\mathrm{NH}$ & $\ldots$ & $\mathrm{NH}$ & $\Delta+1$ & $\Delta+1$ & $\Delta+1$ & $\Delta+1$ & $\Delta+1$ & $\ldots$ & $\Delta+1$ \\
\hline 5 & $\Delta+1$ & $\Delta+1$ & $\mathrm{NH}$ & $\ldots$ & $\mathrm{NH}$ & $\mathrm{H}$ & $\Delta+1$ & $\Delta+1$ & $\Delta+1$ & $\mathrm{H}$ & $\ldots$ & $\Delta+1$ \\
\hline 6 & $\Delta+1$ & $\Delta+1$ & $\mathrm{NH}$ & $\ldots$ & $\mathrm{NH}$ & $\mathrm{H}$ & $\Delta+1$ & $\Delta+1$ & $\Delta+1$ & $\mathrm{H}$ & $\ldots$ & $\Delta+1$ \\
\hline 7 & $\Delta+1$ & $\Delta+1$ & $\mathrm{NH}$ & $\ldots$ & $\mathrm{NH}$ & $\mathrm{H}$ & $\Delta+1$ & $\Delta+1$ & $\Delta+1$ & $\mathrm{H}$ & $\ldots$ & $\Delta+1$ \\
\hline 8 & $\Delta+1$ & $\Delta+1$ & $\mathrm{NH}$ & $\ldots$ & $\mathrm{NH}$ & $\mathrm{H}$ & $\Delta+1$ & $\Delta+1$ & $\Delta+1$ & $\mathrm{H}$ & $\ldots$ & $\Delta+1$ \\
\hline 9 & $\Delta+1$ & $\Delta+1$ & $\mathrm{NH}$ & $\ldots$ & $\mathrm{NH}$ & $\mathrm{H}$ & $\mathrm{H}$ & $\mathrm{H}$ & $\mathrm{H}$ & $\mathrm{H}$ & $\ldots$ & $\mathrm{H}$ \\
\hline 10 & $\Delta+1$ & $\Delta+1$ & $\mathrm{NH}$ & $\ldots$ & $\mathrm{NH}$ & $\mathrm{H}$ & $\Delta+1$ & $\Delta+1$ & $\Delta+1$ & $\mathrm{H}$ & $\ldots$ & $\Delta+1$ \\
\hline 11 & $\Delta+1$ & $\Delta+1$ & $\mathrm{NH}$ & $\ldots$ & $\mathrm{NH}$ & $\mathrm{H}$ & $\mathrm{H}$ & $\mathrm{H}$ & $\mathrm{H}$ & $\mathrm{H}$ & $\ldots$ & $\mathrm{H}$ \\
\hline 12 & $\Delta+1$ & $\Delta+1$ & $\mathrm{NH}$ & $\ldots$ & $\mathrm{NH}$ & $\mathrm{H}$ & $\Delta+1$ & $\Delta+1$ & $\Delta+1$ & $\mathrm{H}$ & $\ldots$ & $\Delta+1$ \\
\hline ... & $\ldots$ & $\ldots$ & $\ldots$ & $\ldots$ & $\ldots$ & $\ldots$ & $\ldots$ & $\ldots$ & $\ldots$ & $\ldots$ & $\ldots$ & $\ldots$ \\
\hline k par & $\Delta+1$ & $\Delta+1$ & $\mathrm{NH}$ & $\ldots$ & $\mathrm{NH}$ & $\mathrm{H}$ & $\Delta+1$ & $\Delta+1$ & $\Delta+1$ & $\mathrm{H}$ & $\ldots$ & $\Delta+1$ \\
\hline$k$ ímpar & $\Delta+1$ & $\Delta+1$ & $\mathrm{NH}$ & $\ldots$ & $\mathrm{NH}$ & $\mathrm{H}$ & $\mathrm{H}$ & $\mathrm{H}$ & $\mathrm{H}$ & $\mathrm{H}$ & $\ldots$ & $\mathrm{H}$ \\
\hline
\end{tabular}

Tabela 2. As contribuições do presente trabalho para coloração total em grafos potência de ciclo. Destacamos as linhas $k=3$ e $k=4$, onde a TCC já estava estabelecida e nós obtivemos a classificação completa. Introduzimos também as novas entradas $H$ e $N H$, representando, respectivamente, os grafos que possuem e os que não possuem coloração harmônica. Note-se a dicotomia obtida para a existência de coloração harmônica Teorema 1.2, evidenciando a dicotomia proposta na Conjectura 1.1.

\section{Compatibilidade e composição de grafos potência de ciclo}

Nesta seção serão definidas operações que possibilitam, a partir de dois grafos já coloridos totalmente $C_{n_{1}}^{k}$ e $C_{n_{2}}^{k}$, colorir totalmente um grafo $C_{n}^{k}$, tal que $n=n_{1}+n_{2}$.

Decompomos um grafo potência de ciclo $C_{n}^{k}$ em dois grafos potência de caminho $P_{n_{1}}^{k}$ e $P_{n_{2}}^{k}$, com $n_{1}+n_{2}=n$, e um conjunto de arestas $S$, tal que é possível usar uma coloração total dos grafos $C_{n_{1}}^{k}$ e $C_{n_{2}}^{k}$ para colorir totalmente $P_{n_{1}}^{k}$ e $P_{n_{2}}^{k}$, respectivamente, utilizando as cores das arestas $E\left(C_{n_{1}}^{k}\right) \backslash E\left(P_{n_{1}}^{k}\right)$ e $E\left(C_{n_{2}}^{k}\right) \backslash E\left(P_{n_{2}}^{k}\right)$ para colorir as arestas de $S$ para obter uma coloração total de $C_{n}^{k}$. As operações serão formalizadas nas Definições 2.1 e 2.2 .

Definição 2.1 (Definição 3.1 da dissertação) $A k$-decomposição em potência de caminho de um grafo potência de ciclo $C_{n}^{k}$ divide o grafo $C_{n}^{k}$ em dois grafos $P_{n_{1}}^{k}$ e $P_{n_{2}}^{k}$, com $n_{1}+n_{2}=n$.

A Figura 1 ilustra uma 3-decomposição em potência de caminho do grafo $C_{18}^{3}$. Podemos notar que é necessário que a distância entre os vértices $w_{x}$ e $w_{y}$ no ciclo do grafo potência de ciclo seja maior ou igual a $2 k$. Além disso, nota-se que todo grafo potência de ciclo $C_{n}^{k} \operatorname{com} n \geqslant 4 k+2$ possui uma $k$-decomposição em potência de caminho.

Definição 2.2 (Definição 3.2 da dissertação) Dizemos que dois grafos $C_{n_{1}}^{k}$ e $C_{n_{2}}^{k}$ são Tipo 1-compatíveis se cada grafo tem uma coloração total Tipo 1 tal que é possível gerar uma coloração total Tipo 1 do grafo $C_{n}^{k}$ utilizando as cores já utilizadas na coloração total de $C_{n_{1}}^{k}$ e $C_{n_{2}}^{k}$.

\section{Todo grafo potência par de ciclo com muitos vértices é Tipo 1}

Na presente seção, mostraremos que todo grafo potência de ciclo $C_{n}^{k}$, com $k$ par e $n \geqslant$ $4 k^{2}+2 k$, é Tipo 1 . 


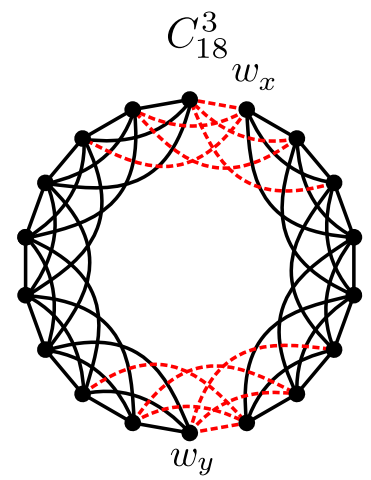

(a)

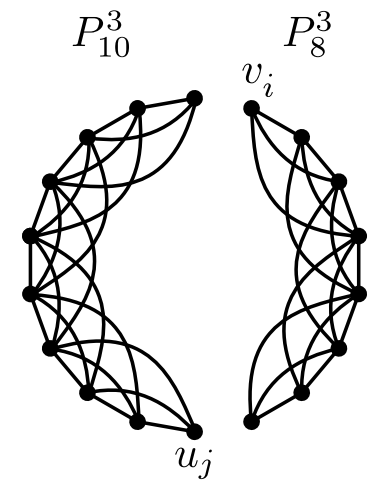

(b)

Figura 1. Uma ilustração do processo de decomposição. (a) 0 grafo $C_{n}^{k}$, com $n=18 \mathbf{e} k=3$. As arestas tracejadas em vermelho representam o conjunto $S$, ou seja, as arestas removidas para formarmos os grafos $P_{n_{1}}^{k}$ e $P_{n_{2}}^{k}$. (b) 0 vértice $v_{i}$ representa o primeiro vértice do grafo $P_{10}^{3}$, o mesmo ocorre com o vértice $u_{j}$ que é o primeiro vértice do grafo $P_{8}^{3}$.

Inicialmente, obtemos no Teorema 3.1 um resultado que demonstra que a Compatibilidade é mantida na operação de composição de grafos potência de ciclo.

Teorema 3.1 (Teorema 3.3 da dissertação) Se dois grafos $C_{n_{1}}^{k} e C_{n_{2}}^{k}$ são Tipo 1compatíveis, então o grafo $C_{n}^{k}$ com $n=n_{1}+n_{2}$ é Tipo 1-compatível com $C_{n_{1}}^{k}$ e com $C_{n_{2}}^{k}$.

No próximo teorema, mostraremos que dois grafos potência de ciclo $C_{2 k+1}^{k}$ e $C_{2 k+2}^{k} \operatorname{com} k$ par são sempre Tipo l-compatíveis. Para tanto, é realizado um procedimento de recoloração a partir do famoso Quadrado Latino $L$ de ordem $2 k+1$, gerando uma coloração total válida representada pela matriz $M$, de ordem $2 k+2$, de tal forma que essa matriz representa uma coloração total Tipo 1 do grafo $C_{2 k+2}^{k}$, que é compatível com a coloração dada pelo Quadrado Latino.

O procedimento é feito em três passos. Primeiramente, a partir da condição harmônica, sabe-se que é necessário trocar as cores de alguns vértices do grafo representado por $L$. Para realizar esta troca utilizaremos o fato de que a matriz $M$ tem células que representam arestas que não existem no grafo $C_{2 k+2}^{k}$. O segundo passo é a inclusão das cores que não existiam em $L$ na matriz $M$. E o último passo é feito para garantir que as duas colorações totais sejam compatíveis.

Combinando o Teorema 3.2 com o Teorema 3.1, mostraremos que todo grafo potência de ciclo $C_{n}^{k}$, com $k$ par, é Tipo 1 , exceto por no máximo $2 k^{2}-k$ grafos, para um dado $k$ fixo.

Teorema 3.2 (Teorema 3.4 da dissertação) Os grafos $C_{2 k+1}^{k}$ e $C_{2 k+2}^{k}$ com $k$ par são Tipo 1-compatíveis.

O Lema 3.3 é utilizado no Corolário 3.4 para mostrar um de nossos principais resultados.

Lema 3.3 (Lema 3.5 da dissertação) $S e z \geqslant a^{2}-a, \exists x, y \geqslant 0$, tal que $z=x a+y(a+1)$.

Nota-se que o limitante mostrado no Lema 3.3 é justo, uma vez que é resultado clássico das equações lineares diofantinas que se $z=a^{2}-a-1$ então $z=x a+y(a+1)$ não possui soluções inteiras válidas. 
Corolário 3.4 (Corolário 3.7 da dissertação) Todo grafo $C_{n}^{k} \operatorname{com} k \geqslant 2$ par e $n \geqslant$ $4 k^{2}+2 k$ é Tipo 1 .

O Corolário 3.4 mostra que para um $k$ par e um valor de $n$ grande o suficiente $\left(n \geqslant 4 k^{2}+2 k\right)$ todo grafo $C_{n}^{k}$ é Tipo 1 . Entretanto, para $k$ par e $n<4 k^{2}+2 k$ obtemos também uma coloração total Tipo 1 para uma série de grafos, mostrando que nossa técnica não atribui uma coloração total ótima para apenas no máximo $2 k^{2}-k$ grafos.

Proposição 3.5 (Proposição 3.8 da dissertação) $S e \quad C_{2 k+1}^{k} \quad e \quad C_{2 k+2}^{k}$ são Tipo 1compatíveis, então no máximo $2 k^{2}-k$ grafos $C_{n}^{k} \operatorname{com} 2 k+1 \leqslant n \leqslant 4 k^{2}+2 k$, não são Tipo 1.

\section{Coloração total em grafos potência de ciclo $C_{n}^{k} \operatorname{com} k=3,4,5$ e 7}

No próximo teorema resolvemos completamente o Problema da Coloração Total para os grafos $C_{n}^{3}$ e $C_{n}^{4}$ para qualquer valor de $n$.

Teorema 4.1 (Teorema 3.9 da dissertação) Um grafo potência de ciclo $C_{n}^{k}$ não completo, com $k \in\{3,4\}$, é Tipo 2 se né ímpar e $k>n / 3-1$, ou Tipo 1 caso contrário.

Os próximos resultados mostram que todos os grafos $C_{n}^{5} \operatorname{com} n \geqslant 110$ e $C_{n}^{7}$ com $n \geqslant 210$ são Tipo 1 .

Teorema 4.2 (Teorema 3.10 da dissertação) Todo grafo $C_{n}^{k} \operatorname{com} k \in\{5,7\}$ e $n \geqslant 4 k^{2}+$ $2 k$ é Tipo 1.

Corolário 4.3 (Proposição 3.11 da dissertação) No máximo 45 grafos $C_{n}^{5} \operatorname{com} n>$ $2 k+1=11$ não são Tipo 1 . E no máximo 91 grafos $C_{n}^{7}$ com $n>2 k+1=15$ não são Tipo 1.

\section{Decomposição de grafos potência de ciclo}

O Teorema 5.1 mostra que todo grafo potência de ciclo com um certo número de vértices ( $n>f(k)$ ) pode ser decomposto em grafos compatíveis de tamanho reduzido. Chamaremos $f(k)$ o número de colorações distintas de $C_{n}^{k}[N[v]]$.

Teorema 5.1 (Teorema 3.12 da dissertação) $S e n>f(k)$, então $C_{n}^{k}$ pode ser decomposto em dois grafos potência de ciclo $C_{n_{1}}^{k}$ e $C_{n_{2}}^{k}$, tal que $n=n_{1}+n_{2}$ e $C_{n_{1}}^{k}$ e $C_{n_{2}}^{k}$ são compativeis.

Se aplicarmos a ideia do Teorema 5.1 recursivamente, podemos gerar uma árvore de composição extremal [Trotignon e Vušković 1994], tal que: a raiz da árvore é o grafo potência de ciclo original, dois nós irmãos são grafos compatíveis, a colagem destes grafos gera o nó pai e todo nó folha é um bloco simples $C_{n_{\ell}}^{k} \operatorname{com} n_{\ell} \leqslant f(k)$. Nota-se que este procedimento é um algoritmo linear para decomposição de um grafo potência de ciclo.

\section{Coloração harmônica para grafos potência de ciclo}

Campos e de Mello mostraram que os grafos potência de ciclo $C_{n}^{k}$, com $n$ ímpar, $k \geqslant 2$ e $2 k+1<n<3(k+1)$, não possuem coloração harmônica. Nesta seção consideramos os demais grafos não completos da classe. E estabelecemos que todos admitem uma coloração harmônica. O Teorema 1.2 segue como corolário do Teorema 6.2. 
Lema 6.1 (Lema 3.13 da dissertação) Seja $C_{n}^{k}$ um grafo potência de ciclo. Se né par e $n \geqslant 4 k+2$, ou se n é ímpar e $n \geqslant 6 k+3$, então $C_{n}^{k}$ possui uma coloração harmônica.

Teorema 6.2 (Teorema 3.14 da dissertação) Seja $C_{n}^{k}$ um grafo potência de ciclo com $2 \leqslant k<\lfloor n / 2\rfloor$. Se $n$ é par, ou se $n$ é ímpar e $n \geqslant 3(k+1)$, então $C_{n}^{k}$ possui uma coloração harmônica.

\section{Impacto do trabalho}

Durante o mestrado, o impacto do meu trabalho em coloração de grafos foi avaliado através de duas publicações em periódico internacional e de três publicações nos anais de conferências internacionais. Participei de três eventos internacionais:

1. Zorzi, A., de Figueiredo, C., Machado, R., Souza, U. S. (2019). Even-power of cycles with many vertices are type 1 total colorable. Electronic Notes in Theoretical Computer Science, 346:747 - 758. LAGOS'2019

2. Zatesko, L., Carmo, R., Guedes, A., Zorzi, A., Machado, R., de Figueiredo, C. (2019). On the chromatic index of complementary prisms. Acta Mathematica Universitatis Comenianae, 88(3):1071-1077. EUROCOMB'2019

3. Zorzi, A., de Figueiredo, C., Machado, R. (2019). An optimal algorithm to totally colorsome powers of cycle graphs. Anais do VI Latin American Workshop on Cliques in Graphs, page 39. LAWGC'2018

$\mathrm{O}$ artigo completo no evento LAWCG foi publicado:

1. Zorzi, A., de Figueiredo, C., Machado, R. (2019). An optimal algorithm to totally color some powers of cycle graphs. Matemática Contemporânea, 46: 175-184.

Os demais artigos completos serão avaliados para os respectivos números especiais nos periódicos Discrete Applied Mathematics e European Journal of Combinatorics. Ainda, durante meu mestrado também participei da elaboração de dois artigos completos, também no tema de coloração:

1. Zorzi, A., Zatesko, L. (2018). On the chromatic index of join graphs and trianglefree graphs with large maximum degree. Discrete Applied Mathematics, 245: 183-189.

2. Zatesko, L., Zorzi, A., Carmo, R., Guedes, A.L.P. (2020). Edge-colouring graphs with bounded local degree sums. Discrete Applied Mathematics, aceito para publicação, https://doi.org/10.1016/j.dam.2020.03.044.

\section{Referências}

Almeida, S. M., Belotti, J. T., Omai, M. M., e Brim, J. F. H. (2014). The total coloring of the 3rd and 4th powers of cycles. Anais do VI Latin American Workshop on Cliques in Graphs, LAWGC'2014, page 32.

Behzad, M. (1965). Graphs and their chromatic numbers. Phd thesis, Michigan State University.

Campos, C. N. e de Mello, C. P. (2007). A result on the total colouring of powers of cycles. Discrete Applied Mathematics, 155:585-597.

Trotignon, N. e Vušković, K. (1994). A structure theorem for graphs with no cycle with an unique chord and its consequences. Journal of Graph Theory, 63:31-67.

Vizing, V. G. (1965). The chromatic class of a multigraph. (em russo). Metody Diskret. Analiz., 3:25-30. 